\title{
Intake power measurement as a criterion for control of HVAC systems
}

\author{
Juliusz Jerzy Walaszczyk ${ }^{1, *}$, Aleksandra Przydróżna ${ }^{2}$, and Edward Przydróżny ${ }^{1}$ \\ ${ }^{1}$ Wrocław University of Science and Technology, Department of Air Conditioning, Heating, Gas \\ Engineering and Air Protection, ul. Norwida 4/6, Building C-6, 50-373 Wrocław, Poland \\ ${ }^{2}$ Max Fordham, St Andrew's House, 59 St Andrew's St, CB2 3BZ, Cambridge, UK
}

\begin{abstract}
Under certain conditions, a HVAC system may consume less energy, provided that the initial response to the increasing heat gains is the increase in the airflow, while cooling is delayed. This ensures that the energy for cooling and reheat is reduced, while the fan power increases. Note that it is difficult to develop the following approach in the standard sequential control algorithms. On the other hand, the use of more complex algorithms than standard sequential algorithms is generally not encouraged because of the increase in the complexity of the control system and its resistance to respond to the varying parameters of a HVAC system. In this work, therefore, to avoid the following complications, the Intake Power Optimization algorithm is proposed. The Intake Power optimization algorithm is compared to the two sequential algorithms: Air conditioning and Airflow priority. To create the reference point enabling comparisons of the three strategies, the optimal control was established using the Nelder-Mead method. It is shown that the Intake power optimization algorithm provides a better control than the sequential algorithms and almost exclusively performs the optimal control actions. As an aside, the results indicate that the same heat gains, however, evenly distributed between rooms may contribute to the significant reduction of the energy demand.
\end{abstract}

\section{Introduction}

Supply Air Temperature (SAT) reset ensures the reduction of the energy demand for cooling and may decrease the energy demand for heating in reheat units. This, however, leads to the increase in the energy demand for air transport $[1,3]$. The primary research problem is, therefore, the pursuit of the optimal air flow rates and the optimal supply air temperatures, ensuring that the benefits of reducing the energy demand for air treatment are higher than the losses caused by the increase in the energy demand for air transport.

Ke \& Mumma (1997) present four methods of controlling the setpoint of the supply air temperature [4]: (i) as a function of the external air temperature; (ii) depending on the heat gains in rooms; (iii) as a function of the air humidity (as a supporting criterion); (iv) the optimal method in context of the energy demand. The majority of publications devoted to

\footnotetext{
*Corresponding author: juliusz.walaszczyk@pwr.edu.pl
} 
the SAT reset control strategy describe setting the supply air temperature as a function of the external air temperature [3-6].

An optimal method of setting the supply air temperature is not obvious [5]. Endhal \& Johansson (2004) compare different control strategies for setting the supply air temperatures to the optimal control. They show a relationship between the total energy consumption (for ventilation) and the supply air temperature. It appears that too low supply air temperature, relative to the optimal value, corresponds to a low risk of a substantial increase in the energy consumption. Simultaneously, too high supply air temperature, relative to the optimal value, may result in a substantial increase in the energy consumption [7]. Besides Ke \& Mumma (1997) state that, the optimization of the primary flow rate and the supply air temperature provides better results in the temperate and dry climate [8].

Diverse SAT reset strategies may be compared, but the optimal control is ultimately of interest. A key research question is: how to select the optimal supply air temperature in the real system? In order to answer this research question, two fundamental issues need to be addressed. First of all, optimization of the supply air temperature often requires working with the model of installation, for instance in methods based on MPC (Model Predictive Control). Note that using a model of HVAC system and performing optimization during standard operation, significantly complicates control system. Second of all, the optimal control is set under defined conditions and usually with constant coefficients of performance. Under real conditions, however, many parameters, which were assumed constant during optimization, are variable, subject to conditions of operation.

\subsection{Research idea (problem)}

The use of more complex algorithms than standard sequential algorithms is generally not encouraged because of the increase in the complexity of the control system and its resistance to respond to the varying parameters of a HVAC system.

In this work, to avoid the following complications, the Intake Power Optimization algorithm is proposed. The algorithm, based on the instantaneous measurement of power intake, generates control actions based on the actual power intake. The approach presented herein avoids the complexity of standard approaches by omitting the necessity to determine the optimal airflow and the optimal supply air temperature. A precise measurement of the airflow, which remains difficult in the existing VAVs [1], is not required in the Intake Power optimization algorithm.

The Intake Power optimization algorithm is compared to the following two sequential algorithms: Air conditioning priority and Airflow priority. Moreover, in order to create a reference point to compare the three strategies to, the optimal control was established using Nelder-Mead method. It allowed estimating the limit of energy savings and establishing under which conditions algorithms provide the optimal control.

\section{Methodology}

All the control strategies were compared, based on the results of simulations performed. Each simulation lasted an hour and was repeated for different external air temperatures in the range 13 to $16.5^{\circ} \mathrm{C}$ with $0.5^{\circ} \mathrm{C}$ step change. Simulations were performed using the computational model of the VAV system, marked as NW3 (Fig. 1). The computational model was calibrated to the nominal parameters (extracted from the documentation for construction). Parameters of the model, which affect the operation in the steady state, were calibrated. Time constants were selected using the method of trial and error. Simulations do not account for air heating in fans. 


\subsection{Heat gains}

The heat gains were related to the number of people within rooms. It was assumed that heat gains are constant in time and linearly dependent on the external air temperature. Two variants of heat gains were considered. The variant A comprised of heat gains that prevented use of reheat units and VAV boxes could be fully open. In other words, in variant A, the air treatment could be limited to the central unit only. In variant B, the sum of heat gains was equivalent to the heat gains in variant A. However, the breakdown of the heat gains within rooms varies, i.e. the room 1.27 has significantly higher heat loads than the two remaining rooms (Fig.1).

Note that the variant A is a special case. Wang et al. (2012) propose the optimal control for an air handling unit with a variable airflow, which services one room [1]. They specify zone 1 , when there is a complete free-cooling, zone 2 , when the airflow is equivalent to the critical value and zone 3 , when the critical airflow is below the minimum flow rate. Using variant $A$ allows investigating the presence of zones 1 to 3 within the modelled NW3 system, even though NW3 services more than one room (three rooms).

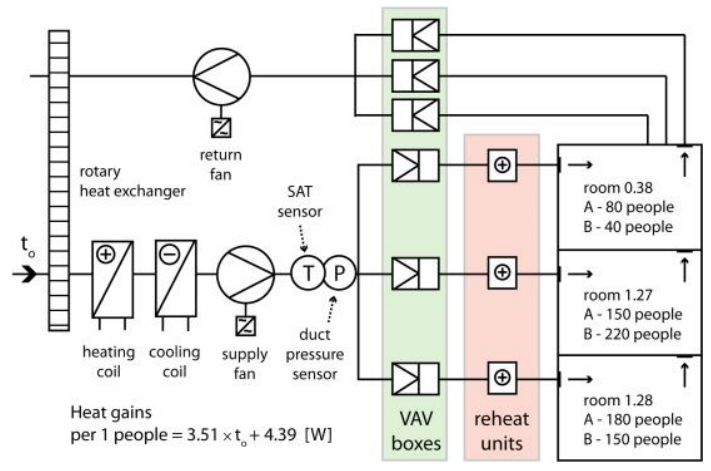

Fig. 1. Schematic of the VAV system NW3 modelled (computationally) in this work.

\subsection{Total energy demand}

All the control algorithms presented in this paper, were compared based on the total energy demand during one hour of operation. Air conditioning priority, Airflow priority, and optimal control actions were investigated under the system's steady state. Once the steady state is established, the total energy consumption may be calculated directly from the power demand (Eq. (1)).

$$
E=P \cdot t
$$

where: $E$ - total energy demand [Wh], $P$ - power demand at steady state [W], $t$ - time [h].

The total power demand is a sum of the power demands of all the components of the VAV system. The fan power $\mathrm{P}_{\mathrm{F}}$ is calculated using Eq. (2).

$$
P_{F}=\Delta p \cdot V / \eta
$$

where: $\Delta p$ - fan pressure $[\mathrm{Pa}], V$ - volume flow rate $\left[\mathrm{m}^{3} / \mathrm{s}\right], \eta$ - fan efficiency. 
The thermal power of each air treatment process in the air handling unit is calculated using Eq. (3).

$$
P_{\text {term }}=V \cdot \rho \cdot c_{p} \cdot \Delta T / \eta_{\text {term }}
$$

where: $\rho$ - air density $\left[\mathrm{kg} / \mathrm{m}^{3}\right], c_{p}$ - specific heat $[\mathrm{J} /(\mathrm{kg} \cdot \mathrm{K})], \Delta T$ - temperature difference at the inlet and outlet of the device $[\mathrm{K}], \eta_{\text {term }}$ - normalized thermal power coefficient.

The value of coefficient $\eta_{\text {term }}$ is estimated from the thermal energy cost and the cost of equivalent electric energy (Eq. (4)). Normalization was performed in order to compare the electric energy and thermal energy.

$$
\eta_{\text {term }}=\text { electric energy cost / thermal energy cost }
$$

\section{Control algorithms}

The control of fan speed, as a function of constant pressure, is not energy efficient. The setpoint is usually selected, based on the peak-load, even though the majority of operation is under lower loads [2]. For this reason, all algorithms (Air conditioning priority, Airflow priority and Intake power optimization) have the duct static pressure reset. Simulations were carried out with the assumption that at least one of the VAV dampers was almost fully open.

\subsection{Air conditioning priority}

The sequential control with the priority set on the air treatment, initially controls the efficiency of a cooling coil, in order to maintain the design conditions within rooms. When the supply air temperature is greater than or equal to the minimum supply air temperature, the control system regulates the operation of the cooling coil, while maintaining the minimum airflow. A further increase in the heat gains leads to maintaining the minimum supply temperature and controlling the volume flow rate. Fig. 2 shows examples of distribution of the control sequence. Each room has an individual PID regulator. The maximum signal from all the regulators controls the efficiency of devices in the air handling unit. The following approach allows cooling the air to the requirements of the room with the highest heat gains. The supply air temperature in the other rooms with lower heat loads may be adjusted in the local reheat units.

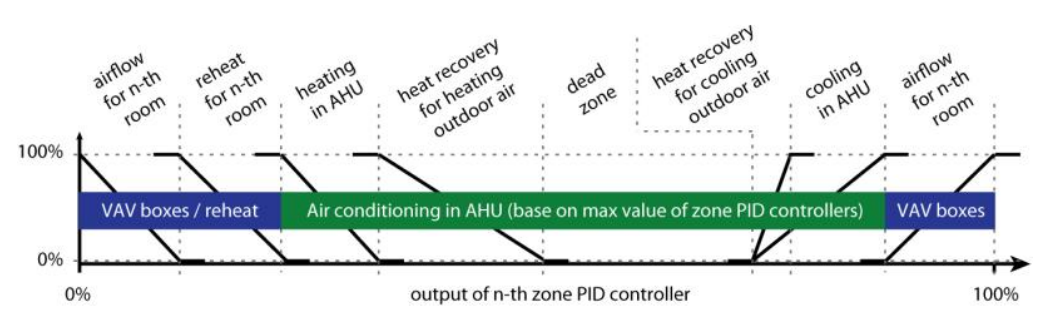

Fig. 2. Control sequences for Air conditioning priority strategy. 


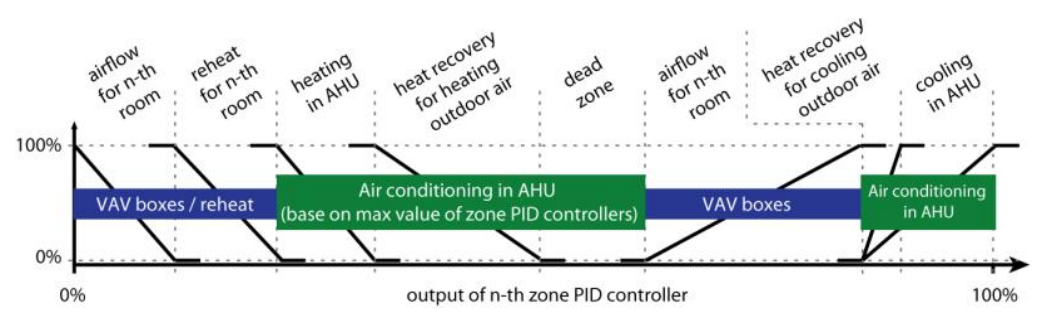

Fig. 3. Control sequences for Airflow priority strategy.

\subsection{Airflow priority}

The sequential control with priority set on the airflow, initially controls the volume flow rate in order to maintain the design parameters in rooms. When the maximum airflow is supplied to the room, the control system begins cooling in the air handling unit. The sequences of control in the Airflow priority algorithm are shown in Fig. 3.

\subsection{Intake power optimization}

The air temperature outside the air handling unit is set within limits of the acceptable supply air temperature, based on the instantaneous measurements of power intake in the ventilation system. The algorithm actions may be presented in four steps. Each step has its individual, constant time of operation. Step 1 (modify): the algorithm slightly modifies the setpoint temperature outside the central unit, for example by $+0.1^{\circ} \mathrm{C}$. Step 2 (wait): the algorithm waits for the system to operate in steady state (corresponding to the set values of the supply air temperature and the airflow). In this version of the algorithm reaching the steady state is not verified and step 2 is treated as a safety margin only. Step 3 (measuring power): the control system measures the instantaneous power intake and averages the value over set time. Step 4 (make decision): the algorithm makes a decision regarding the direction of changes. If the initial modification $\left(+0.1^{\circ} \mathrm{C}\right)$ results in the decrease in power intake, the control system will try to introduce the same type of modification. If the initial modification results in the increase in power intake, the control system will reverse the direction of changes to $-0.1^{\circ} \mathrm{C}$.

The Intake power optimization strategy is characterized by the variable supply air temperature setpoint. The chiller power and the fan power vary as well. In case of the Intake power optimization strategy, the instantaneous power intake must be integrated with respect to the time. 


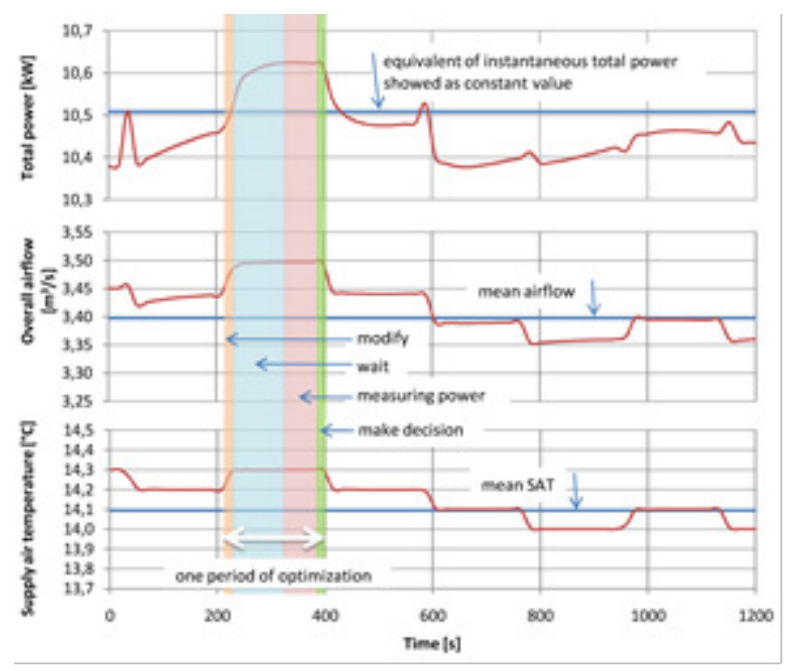

Fig. 4. Total power, overall airflow and SAT charts for Intake power optimization strategy.

\subsection{Optimal control actions}

The set of optimal control actions was used as a benchmark for the Intake power optimization and the sequential control strategies. Nelder-Mead simplex method, implemented in the Matlab function fminsearch, was used on the function $\mathrm{P}_{\mathrm{HVAC}}$ (Eq. (5)).

$$
P_{H V A C}\left(\Delta p_{S D} ; \Delta p_{R D} ; t_{o} ; V_{1} ; V_{2} ; V_{3}\right)=P_{\text {term } C C}+P_{\text {termRI }}+P_{\text {termR } 2}+P_{\text {termR3 }}+P_{S F}+P_{R F}
$$

where: $P_{H V A C}$ - total system power [W], $\Delta p_{S D}\left(\Delta p_{R D}\right)$ - supply (return) duct static pressure $[\mathrm{Pa}], t_{o}$ - external air temperature $\left[{ }^{\circ} \mathrm{C}\right], V_{l}\left(V_{2}, V_{3}\right)$ - supply airflow to the room $0.38(1.27$, $1.28)\left[\mathrm{m}^{3} / \mathrm{s}\right], P_{\text {term CC }}$ - cooling power $[\mathrm{W}], P_{\text {termRI }}\left(P_{\text {termR2 }}, P_{\text {termR } 3}\right)$ - reheat power for room $0.38(1.27,1.28)[\mathrm{W}], P_{S F}\left(P_{R F}\right)-$ supply (return) fan power [W].

\section{Results}

\subsection{Intake power optimization works in non-steady state}

With constant heat gains, both the sequential algorithms (Air conditioning priority, Airflow priority) and the optimal control actions, which were determined during optimization, operate in the steady state. The proposed algorithm (Intake power optimization), however, does not operate in the steady state. This is caused by continuous changes in time of the supply air temperature and the airflow. As a result, the control signals fluctuate about the optimal control setpoints. Fig. 4. shows example results of simulation, where the external air temperature was set at $14.5^{\circ} \mathrm{C}$. The duration of step 2 (wait) was selected with a safety margin, but at some instances, a HVAC system fails to reach a steady state. The results of simulation indicate that this should not be an issue. Similarly, the algorithm oscillates between the optimal control actions but with a greater standard deviation than the mean supply air temperature. 


\subsection{Compare control strategy}

The air conditioning priority ensures HVAC operation with the minimum airflow that allows assimilation of heat gains within rooms (upper graph in Fig. 5 - 6). As a result, this algorithm contributes to the maximum reduction of energy consumption by fans (Fig. 8 and 11). Based on the results of simulation, it appears that the Air conditioning priority algorithm has a greater total energy demand for the optimal control, particularly at lower external air temperatures. For instance, in variant $\mathrm{B}$, at $14^{\circ} \mathrm{C}$, optimal control actions are responsible for the energy consumption $20 \%$ lower than the Air conditioning priority (Fig. 12).

The Airflow priority algorithm ensures HVAC operation with relatively low energy consumption for air treatment (Fig. 7 and 10). The reduced energy demand for air treatment, however, corresponds to a very high energy demand for fans, compared to the other algorithms (Fig. 8 and 11). For instance, in variant $\mathrm{A}$, at $16^{\circ} \mathrm{C}$, the optimal control actions account for $80 \%$ less fan power compared to the Airflow priority (Fig. 8).

The Intake power optimization algorithm has a similar energy demand to the optimal control. Based on the following, the optimal control algorithm must have both a static pressure reset and SAT reset.

\subsection{Different profiles of heat gains}

There are two aspects worth discussing when considering variants of distribution of heat gains. First of all, there is an aspect of energy conservation. Note that the same heat gains, however, evenly distributed between rooms may contribute to the significant reduction of energy demand. For instance, in variant $\mathrm{B}$, at $13^{\circ} \mathrm{C}$, an hour of optimal control uses $8.8 \mathrm{kWh}$, whereas, in variant $\mathrm{A}, 2.7 \mathrm{kWh}(31 \%)$. Similar trends are observed at different setpoint temperatures. For instance, at $16.5^{\circ} \mathrm{C}$, an hour of optimal control uses $17.7 \mathrm{kWh}$ in variant $\mathrm{B}$ and $12.2 \mathrm{kWh}(69 \%)$ in variant $\mathrm{A}$.

The second aspect corresponds to the comparability of the control algorithms. The more unevenly distributed heat gains between rooms are, the less apparent are the differences between the different algorithms. In variant $\mathrm{A}$, the differences between the minimum and maximum total energy demand were in the range 0 to $11.6 \mathrm{kWh}$, whereas in variant $\mathrm{B}$ these were in the range 0.6 to $2.6 \mathrm{kWh}$. 


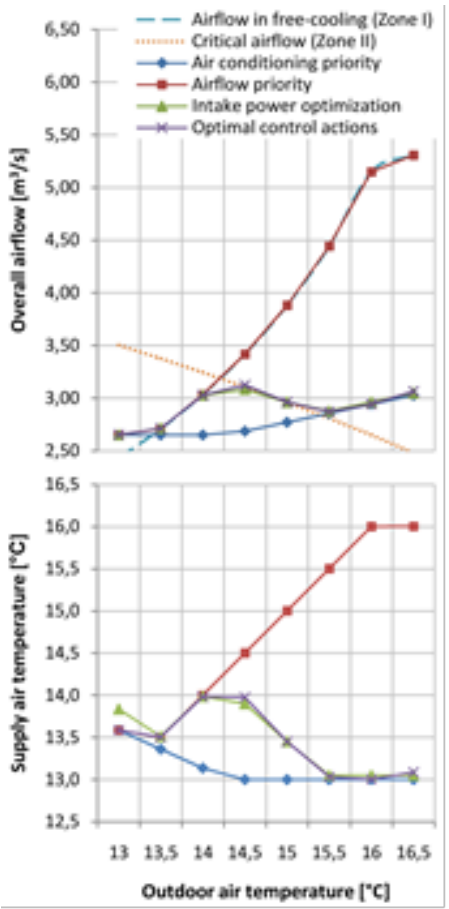

Fig. 5. Overall airflow and SAT by heat gains profile in variant A.

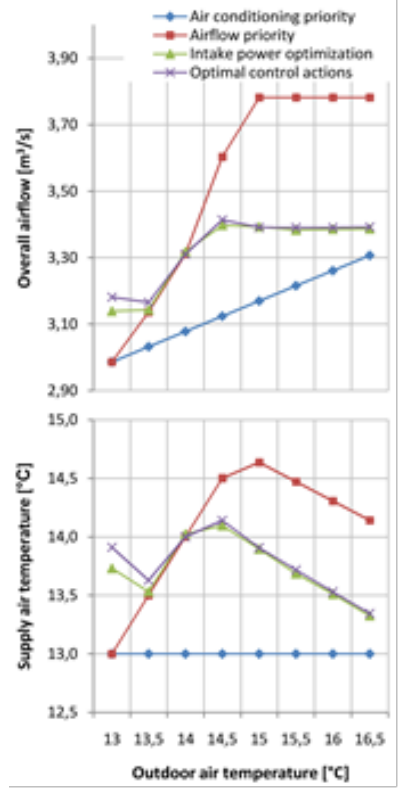

Fig. 6. Overall airflow and SAT by heat gains profile in variant B. 


\subsection{One room effect}

In [1] Wang et al. (2012) used analytical methods to determine the optimal control for a system servicing one room. They showed relationships that allowed performing the optimal control actions during the economizer strategies. The analytical determination of the optimal control in the remaining modes of operation of ventilation allows to prepare a complete 'control table', which displays the optimal control under any conditions. It is shown that, in variant A, VAV system with three rooms is simplified to one room. As a result, the same control table may be used to determine the optimal control for a more complex system, consisting of more than one room. This, however, applies to the heat gains in variant A. Fig. 5 shows the total supply airflow and the supply air temperature in variant A. Additionally, the optimal airflows, determined analytically for one room (Zone I, Zone II) are marked in Fig. 5. The Intake power optimization algorithm ensures the optimal control because it 'goes correctly' through Zone I and Zone II.

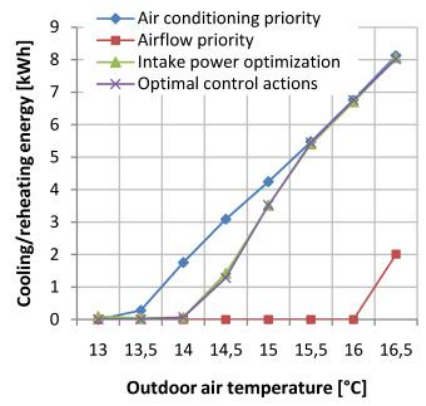

Fig. 7. Cooling/reheating energy in variant $\mathrm{A}$.

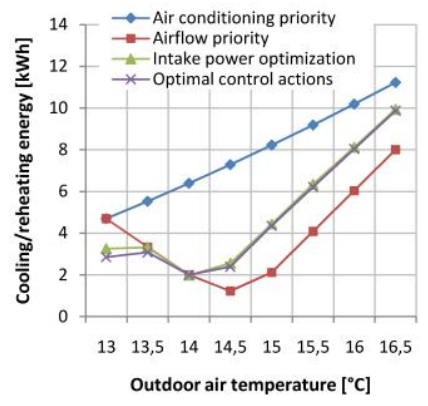

Fig. 10. Cooling/reheating energy in variant $\mathrm{B}$.

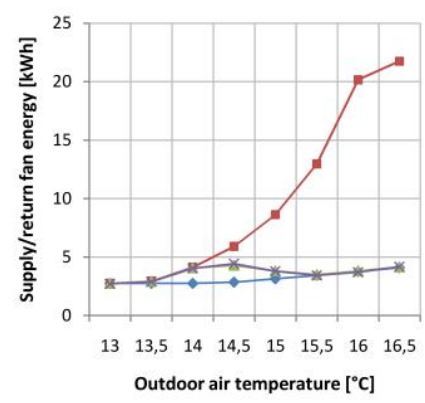

Fig. 8. Fans energy in variant $\mathrm{A}$.

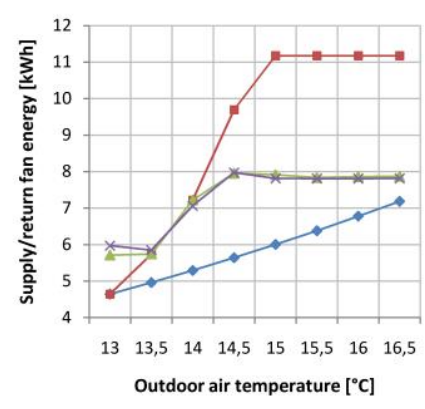

Fig. 11. Fans energy in variant $\mathrm{B}$.

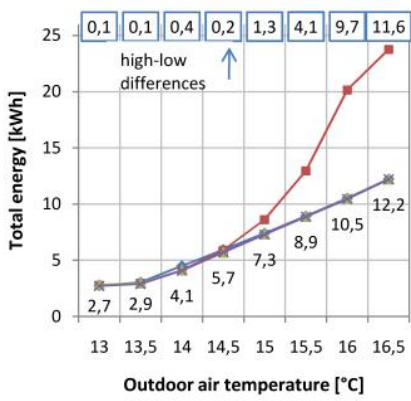

Fig. 9. Total energy in variant $\mathrm{A}$.

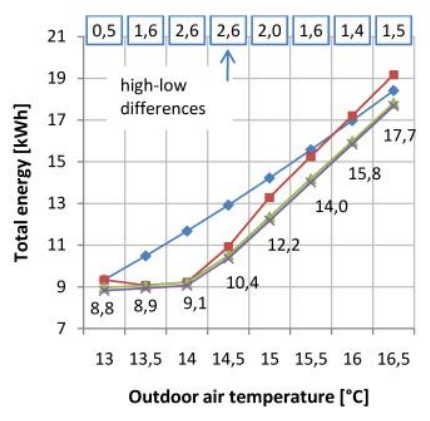

Fig. 12. Total energy in variant $\mathrm{B}$.

It appears that, by calculating the optimal control for a system that services one room only, the lower limit of the energy demand of a more complex system may be established. This lower limit could be used as a criterion to evaluate the energy consumption of ventilation system in practice. If the control algorithm implemented controls the system closely to the optimum, then based on the calculated limit, the entire system can be evaluated. For instance, if the ventilation system operates consuming significantly more energy than the lower limit value, it may indicate that the system was designed for different profiles of heat gains. To avoid too high energy consumption, the following two actions could be undertaken, either the system could be modernised or the use of rooms could be modified to ensure more energy efficient use of the system. 


\section{Conclusions}

In the presented examples, the Intake power optimization algorithm provides a better control than the sequential algorithms and almost entirely performs optimal control actions. It may be deduced that implementing the duct static pressure reset and the SAT reset in the control system strategy enables the optimal control.

Implementing a SAT reset in a real ventilation system is not easy. Control strategies using the model are quite complex and sensitive to the assumed values of coefficients (e.g. efficiency). Therefore, the Intake power optimization strategy is based on measuring the actual power intake of the system. In this way, the complex issue of choosing the optimum control action was modified to the question of proper measurement.

Based on the simulations performed, the performance of Airflow priority algorithm was poor, particularly in variant A. Note that the increase in the supply air temperature to the maximum level is equivalent to the application of the Airflow priority algorithm. This effect would, thus, be negative. As a result, while implementing the SAT reset strategy, the margin of error should be set towards the lower temperatures. In other words, it is better to set the low setpoint supply air temperature than too high. Similar conclusions are drawn in $[5,8]$.

Moreover, the performed simulations indicate that calculating the optimal control for the system servicing one room only may be used to determine the lower limit of energy demand for a more complex system. This limit may be used as a criterion to evaluate the energy consumption of ventilation system in practice. The lower limit allows assessing if the system is designed for the profiles of heat gains that occur most often and if the system is controlled optimally.

The research to follow should be focused on the implementation of the Intake power optimization algorithm in real buildings. First of all, the measurement of power intake could be based on the electric meter, provided that the devices installed are charged with electric energy only. The measurement of the total power intake by chillers operating cyclically and then translating it into the constant value in time could be challenging.

\section{References}

1. G. Wang, L. Song, Energy and Building 49, 310-316 (2012)

2. G. Liu, M. Liu, ASHRAE Transactions NY-08-055, 451-457 (2008)

3. G.S. Okochi, Y. Yao, Renewable and Sustainable Energy Reviews 59, 784-817 (2016)

4. Y. Ke, S.A. Mumma, Energy 22, 601-614 (1997)

5. Energy Design Resources, The advanced variable air volume (VAV) system design guide (2009)

6. J. Murphy, Ashrae Journal, October, 18-28 (2011)

7. F. Engdahl, D. Johansson, Energy and Buildings 36, 205-218 (2004)

8. Y. Ke, S.A. Mumma, International Journal of Energy Research 23, 371-387 (1999) 